Horizons philosophiques

\title{
Nouvelles technologies de l'image et démocratie
}

\section{Marie-José Mondzain}

Volume 11, numéro 1, automne 2000

L’amodernité de la photographie?

URI : https://id.erudit.org/iderudit/802947ar

DOI : https://doi.org/10.7202/802947ar

Aller au sommaire du numéro

Éditeur(s)

Collège Édouard-Montpetit

ISSN

1181-9227 (imprimé)

1920-2954 (numérique)

Découvrir la revue

Citer cet article

Mondzain, M.-J. (2000). Nouvelles technologies de l'image et démocratie. Horizons philosophiques, 11(1), 9-24. https://doi.org/10.7202/802947ar d'utilisation que vous pouvez consulter en ligne.

https://apropos.erudit.org/fr/usagers/politique-dutilisation/ 


\section{NOUVELLES TECHNOLOGIES DE L'IMAGE ET DÉMOCRATIE}

Quels rapports l'image entretient-elle avec la liberté dans une communauté politique? On a parlé d'emblée de démocratie pour annoncer cette rencontre. Je ne sais ce que c'est, sauf à en donner quelques définitions minimales, par exemple dire qu'on désigne par ce terme le régime d'une communauté où l'on est libre de se battre pour la liberté sans courir un danger vital, ou bien encore la définir sur un mode plus universel par le respect de droits valables pour tous sur l'ensemble de la planète. Ces définitions approchées pourraient ouvrir tous les débats du type «informatique et liberté». Mais ici, il ne sera question que d'image, c'est pourquoi je choisirai une autre formulation en disant qu'un régime est démocratique quand il fait de la vérité le produit de la parole échangée par des sujets en vue de leur cohabitation, et non le résultat doctrinal ou scientifique d'une logique du tiers exclu. Autrement dit, dans les dictatures du corps ou sous le joug de l'autorité de l'esprit, de deux propositions contradictoires, si l'une est vraie l'autre est nécessairement fausse. Dans la communauté des citoyens, c'est justement la place du tiers qui a un effet de vérité. C'est justement là que l'image a établi ses quartiers. La démocratie n'a rien à voir avec une liberté considérée comme acquise, mais avec la possibilité de maintenir un débat critique, une mobilisation de la parole. Ce qui est démocratique, c'est la culture du désaccord et la recherche d'un accord. Si je pose ainsi la question en mettant en avant le régime non binaire, non disjonctif de la liberté des sujets qui partagent un même monde, c'est parce que, comme je vais essayer de le démontrer, l'image a un rôle constitutif dans la construction de ce dispositif du tiers inclus.

Au cœur des questions que nous nous posons sur les menaces qui pèsent aujourd'hui sur notre liberté, les productions visuelles d'origine électronique ont-elles une responsabilité singulière? Portentelles atteinte à la vitalité critique des regards dans la communauté? Les nouvelles techniques, mises au service des visibilités, c'est-à-dire l'ensemble des possibilités offertes par les ordinateurs et les mises en réseau, sont-elles coupables de dictature? Ou bien faut-il déplacer la question, c'est-à-dire considérer une technique pour ce qu'elle est, un instrument et rien de plus, et prendre en compte les dispositifs actuels du marché de la communication et de la consommation du visible 
comme le lieu véritable des enjeux de la liberté? Cependant, accuser le marché d'entrée de jeu serait trop simple et réduirait notre réflexion à une déploration contre l'empire de Microsoft. Ce n'est pas ma position. Au contraire, la philosophie doit se soucier d'abord de définitions et la pensée politique formuler ses exigences : alors seulement, nous pourrons désigner les véritables adversaires de la liberté. Interroger l'image et interroger la technique, quand leurs destins sont comme aujourd'hui étroitement mêlés, sont deux moments différents du questionnement. C'est pourquoi ma réflexion se fera en deux temps : un retour historique sur l'image et la liberté, une mise en perspective de ces données avec les techniques actuelles mises au service des visibilités.

C'est sur la notion même d'image qu'il faut revenir afin de savoir si les nouvelles techniques produisent ou non des images, et s'il peut arriver que l'inflation du visible anéantisse l'image purement et simplement, pour quelles raisons et de quelle façon.

Toutes les images faites de mains d'hommes sont artificielles et depuis toujours résultent d'une technique productive. Je laisse aux médiologues le soin de réduire les effets d'un produit aux procédures de sa production. Cela risque de conduire à une rhétorique maniacodépressive sur l'avant et l'après. Je préfère considérer la vitalité du présent. Les choses de notre civilisation étant hic et nunc, ce qu'elles sont, coprésentes à nos vies et déterminant notre cohabitation, il nous incombe tout simplement de trouver les moyens d'en faire une culture. Une culture, c'est-à-dire un ensemble de choses matérielles et symboliques susceptibles de donner ses chances et son sens au partage d'un même monde. Une image appartient au monde sensible, et nous redoutons l'insensibilité des machines, oubliant que le miroir de Dürer n'était pas plus animé qu'un ordinateur, même plutôt moins.

\section{A) L'image est liberté, la dictature hait l'image.}

L'entrée de l'image dans notre culture s'est opérée sous le signe de la liberté et du débat politique. L'image est née sous l'emblème de l'ambivalence, de l'ambiguitté, puisqu'elle arrive avec le récit de l'incarnation et de la passion dans les mains de ceux qui en firent un enjeu de pouvoir. Elle est liberté et elle est pouvoir, cela dès les origines de son introduction officielle dans notre monde. Rien n'a changé de ce côté-là, même si la distribution de la liberté et du pouvoir se trouve totalement transformée à chaque grand moment de l'histoire matérielle et symbolique. 
Je vais d'abord tenter de cerner ce qu'est une image, c'est-à-dire pas grand-chose avec de grands effets. Ensuite, voir l'ambivalence historique de sa gestion, et cela afin de pouvoir interroger les nouvelles techniques et leur compatibilité avec une vitalité de la liberté et du jugement dans une communauté toujours soucieuse de se préserver des dictatures.

L'interdiction portant sur la fabrication des images aussi bien que le régime de disqualification qu'elles subissaient avaient deux raisons fondamentales qui nous enseignent par voie de conséquence ce qui se joue dans la production des visibilités. Dans la pensée théologique juive, l'image est impure et sa souillure indissociable de celle du sang. Ainsi peut se comprendre que l'image soit du côté du mélange de la chair et du sang, de toutes les turbulences de la vie donc de la mortalité. L'autre obstacle à l'intronisation des images vient de la métaphysique : son absence de gravité ontologique, son manque de réalité substantielle. Tout la condamne à n'être qu'un objet douteux et illusoire qui fait sombrer les esprits dans l'erreur et le mensonge.

\section{1) La victoire de l'impur}

La fabrique de l'image est une invention du christianisme paulinien, c'est même sans doute son invention la plus fondatrice si l'on juge qu'en dépit de l'affaiblissement progressif des valeurs strictement religieuses - qui ne va pas sans le retour des revendications spirituelles -, l'interprétation de l'incarnation comme synonyme de l'introduction légitime des images, non seulement résiste, mais est devenue une figure centrale de la modernité. Le legs du nouveau testament, c'est l'image, sous le double signe et de la liberté et de la dictature.

Si dans la perspective biblique l'image est interdite à la fabrication, c'est parce qu'elle doit rester pure, sans mélange, invisible. Image coupée du sang et de toutes les souillures qui maculent, elle est pure, sainte, et sans tache. Personne ne la voit, personne ne peut la faire. Seul le dieu unique a le droit de produire une créature ayant titre d'image. La seule occasion pour nous de la voir, dans la créature, est anéantie par la chute et nos yeux sont pour toujours privés du spectacle de notre similitude. L'image visible est donc déterminée par un écart infranchissable qui la sépare de toute essence, de toute transcendance. Sa sacralisation est construite sur le déni de son inconsistance. 
La levée de l'interdit de fabrication inaugure l'entrée royale de la souillure, du mélange et des taches, par la voie de l'image, dans la culture occidentale. L'universalisme paulinien est la première formulation de la mondialisation des visibilités. Cela se fit sous le signe de l'incarnation, de l'économie passionnelle, elle devient l'objet de la gestion et de l'administration de nos émotions par une institution qui, riche de la libération du visible, se soucie aussitôt d'en prendre le contrôle.

Dire qu'une image incarne, dans le champ du visible, la figure d'une invisibilité, la définit comme incarnation de cet écart infranchissable avec la présence ou la substance de son référent. Pas d'image sans une absence. L'élimination du sang était conjuration des menaces fusionnelles, sans doute incestueuses. L'introduction de la maculation sanglante du visible doit malgré tout maintenir l'interdit fusionnel. L'image est ennemie de l'un, faute de quoi elle est une idole. Une image ne sera donc que la semblance, l'apparition d'une figure qui fait signe à celui qui la regarde, sans jamais combler son désir de voir par une réelle présence dans une identification substantielle. Cette situation du regard est la fondation de la liberté de ce regard qui déploie toute la mobilité de son jugement sur l'objet de son désir de voir. Une image ne matérialise rien, ne personnifie rien, ne prend pas corps et ne prend pas les corps. Désignée comme le contraire d'une idole par le fait même de la vacuité qui la soutient, une image n'est rien de plus qu'une image ou comme dit Jean-Luc Godard "pas une image juste, juste une image". L'image sans gravité ouvre à la gravité d'une autre question, celle de la responsabilité du regard et de la pensée de ceux qui les produisent et les reçoivent.

Durant la querelle qui opposa les partisans de l'image à ceux qui voulaient la détruire, c'est sur ce point que le débat se dénoua au profit des iconophiles. C'était de leur côté que l'image était reconnue comme chose sans substance, et sans poids, dans une disponibilité absolue. Il était clair, pour un partisan de l'image, qu'en aucun cas elle ne pouvait prétendre à l'être ou à la vérité et que l'inconsistance intrinsèque qui est sa marque, fait de son incarnation un lieu de mouvement, de motion. C'est aux mains qui la fabriquent et aux yeux qui la regardent d'en répondre. Née sous le signe du mélange sanglant de la chair et de la vie, l'image tombe sous la responsabilité de ceux qui gèrent à travers elle le destin de leurs émotions. Ainsi les iconophiles pouvaient-ils dire à leurs adversaires que c'est parce qu'ils considèrent l'image avec des yeux d'idolâtres qu'ils n'y voient 
qu'une idole. Mais pour qui pose sur elle un regard plein de grâce, le même objet bascule tour à tour de l'idole à l'icône. Jouissant de la présomption d'innocence, elle appelle la construction du regard. Indécidable et travaillée par l'ombre, elle flotte dans un intermonde dans l'attente de notre parole et de nos choix.

Rejeton du désir de voir qu'elle ne vient jamais combler, elle ne communique rien d'autre qu'un mouvement, celui de la pensée et le jugement libre. Le jugement désigne ici un acte et non l'énoncé d'un verdict.

\section{2) La liberté du jugement}

L'autre révolution spéculative qui entama, dans l'Antiquité, les préventions contre l'image fut sans aucun doute la pensée sophistique, celle que Socrate aussi bien que Gorgias, mais de façon bien différente, avaient pratiquée quelques siècles plus tôt dans la cité. II est vrai que la dignité ontologique de l'héritage platonicien et de la métaphysique aristotélicienne, totalement soucieux de préserver l'unité de l'être et du vrai, ne pouvait que réserver à l'image un sort disqualifié. Pour la philosophie, dans son apparition sensible, le monde est composé d'ombres illusoires dont il faut nous défaire pour entretenir un rapport apodictique avec l'être et la vérité. Cet héritage métaphysique est aussi lourd que put l'être la disjonction du pur et de l'impur dans la pensée juive. La disjonction de l'être et du non-être, du vrai et du faux, dominée par le principe propositionnel du tiers exclu, met l'image dans une posture irrecevable, elle qui participe des apparences d'un monde périssable dépourvu d'être et d'éternité. Son incertitude exige qu'on la rejette ou qu'on en fasse un intermédiaire métaphorique et transitoire sur le chemin qui conduit à l'un. L'un est ennemi de l'image tout comme la pureté. Impossible dans ces conditions de trouver en elle un principe unifiant. L'image substantielle n'existe pas plus que l'image unifiée, il n'y aura jamais d'image vraie ni d'image du vrai, et l'image est l'objet antiphilosophique par excellence. Entendons qu'elle ne peut être qu'un objet politique, objet de la convocation au débat contradictoire et au choix commun. Autrement dit, on a les images qu'on mérite, ou si l'on préfère, comme dit Hannah Arendt, la communauté des hommes qui vivent ensemble choisit les figures de la culture qui dessinent sa conception commune du sens et de la liberté. Elle appelle cela : "choisir ses compagnons parmi les hommes, les choses, les pensées, dans le présent comme dans le passé". C'est pourquoi elle dit encore que l'artiste n'est contraint par rien, pas même par la vérité. J'insisterai à mon tour pour 
dire que l'image est par excellence ce qui échappe à la vérité, qu'elle soit métaphysique, scientifique ou révélée. Qu'on n'aille pas surtout en conclure que je dis que l'image est fausse ou encore mensongère, cela reviendrait à lui appliquer a contrario les critères dont elle est indépendante. Mieux vaut dire que la vérité n'est pas le problème de l'image, c'est le problème du jugement qui face à elle donne un sens, donc si l'on veut une valeur de vérité à la mobilisation du jugement. Cette valeur n'est ni morale, ni religieuse, ni scientifique, puisqu'elle n'a d'autre critère que la forme de liberté qu'on choisit de partager. On retrouve une dimension évoquée précédemment à savoir que la vérité de l'image est sous la responsabilité du sens que le jugement décide de lui donner. Cette responsabilité politique ne renvoie pas à la morale mais à l'éthique. Par quoi j'indique qu'elle ne fait pas appel à la conscience du bien et du mal, mais à la responsabilité. Si l'image n'est ni vraie ni fausse, ni bonne ni mauvaise, cela signifie qu'elle se juge à l'aune de la place qu'elle fait au tiers à qui elle s'adresse. Elle n'impose rien, elle n'engloutit pas, elle produit de l'altérité.

Autant dire qu'elle est tout entière prise sous le régime de l'opinion que les Grecs appelaient la doxa. L'image est le lieu du débat contradictoire, lieu du probable et de l'incertain, comme toutes ces choses dont les hommes qui vivent ensemble discutent pour choisir ce qui donne sens à leur rassemblement. Nous approchons là quelque chose qui ressemble à l'idée de la démocratie, si l'on veut bien désigner par là le régime intersubjectif du croisement des signes dans un espace commun. L'image a donc bien un statut sophistique, au sens où c'est sous ce régime de la parole dans la cité, que la philosophie dut reconnaître les limites de son autorité spéculative et de ses utopies. Je renvoie ici au beau travail de Barbara Cassin, qui m'a aidée à mieux saisir l'enjeu politique de la circulation des discours quand ils traitent des matières incertaines, de la justice et du pouvoir, de tout ce qui tisse l'histoire réelle des hommes dans leur cohabitation. Image, liberté, démocratie ne sont que des mots livrés à la responsabilité des hommes en quête d'humanité.

L'indécision de l'image, son indécidabilité aussi longtemps qu'elle est désertée par la parole la met dans nos mains dans une situation d'innocence, de mélange douteux, de turbulence équivoque.

Si l'on accepte ce que je viens de dire, alors se pose la question suivante : nos nouvelles images prendraient-elles le double risque d'être pures et univoques? 


\section{3) Les menaces de la communication}

Pour y voir plus clair il faut en passer par une autre donnée. Puisque j'ai à ce point lié l'intronisation de l'image à l'incarnation et à la liberté, je ne peux faire l'économie d'une réalité institutionnelle d'origine encore ecclésiale, tout aussi présente. Je veux dire que l'institution s'est emparée immédiatement du visible dans l'illustration stratégique et pédagogique de ses programmes de conquêtes. Paradoxalement, en effet, l'instrument de la liberté et du débat, a été mis en quelques siècles au service de la messagerie universelle. Puisque l'image avait cette force émotive et persuasive que donne la présence d'un spectacle, il ne pouvait être question de la laisser se développer sans contrôle et de ne pas utiliser sa puissance pour en faire le privilège exclusif de ceux qui en garderaient le monopole. La liberté soit, mais pas pour tous. Comme disait saint Paul : "Désormais tout est permis mais tout ne m'est pas profitable". L'image est devenue le mode de transmission, de diffusion et de communication univoque de tous les dispositifs de pouvoir. De la communication du mouvement on est passé à la communication tout court. Elle est devenue représentation d'un corps incorporant tous les corps. Définie par son équivalence avec le message doctrinal, on lui reconnut non seulement des privilèges supérieurs à ceux de la parole, mais on la chargea de réduire cette parole au silence : s'adresser directement à la croyance, combler les yeux et franchir la barrière des langues, comme un espéranto planétaire, elle fut ni plus ni moins appelée "Bible des illettrés". Dans un programme de communication aujourd'hui millénaire, l'image fut chargée de dire la seule vérité acceptable et de répandre des croyances irréfutables qui ne laissent plus la moindre place à l'indécidabilité opinative du visible. Instrument du silence et de la soumission, les visibilités quittèrent la pensée de l'image pour la stratégie du visible. Construite pour diffuser une pensée unique fondée sur l'exclusion de ceux qui ne s'y soumettaient pas, elle se fit univoque et impérative. La communication réinstalle inévitablement un binarisme disjonctif, une logique du tiers exclu qui ramènent les visibilités à une imagerie illustrative et une rhétorique démonstrative. Les images sont là pour faire savoir et pour faire croire et sont décidées a priori par ceux qui ne souhaitent pas qu'une image donne à penser donc à parler. La catéchèse, la propagande et la publicité, toutes, suivent cet usage programmatique et informationnel du visible afin de faire consommer et digérer des programmes dont le modèle n'est pas loin d'être eucharistique. C'est ici que la pensée 
chrétienne fournit encore les clés de la lecture. C'en est fait de l'écart et de l'altérité; on produit des visibilités fusionnelles, offertes à la consommation communielle du vrai. La consommation aujourd'hui n'est plus un rite mais l'objet d'un marché, celui de la manducation planétaire d'une nourriture universelle : en mangeant le corps du visible, nous devenons membres de ce corps. Et comme on ne peut pas parler la bouche pleine, tous les corps digèrent en silence des objets qui ne s'offrent plus à l'indécision contradictoire où s'originent les débats et les choix. La communication a un bien vieux modèle, c'est la communion. "Hoc est corpus" se dit désormais "Hoc est imago".

C'est ainsi que le sacrement de l'information, le culte de la communication, et les dispositifs consensuels de la communion ont été inaugurés par ceux là même qui avaient ouvert le champ le plus vivant de notre liberté critique. Lesquels, comme on sait, sont tout à fait à l'aise dans les nouveaux media. Cette situation mit définitivement le visible en crise, je veux dire dans un déchirement ininterrompu entre le régime de l'asservissement mercantile et celui de la liberté intersubjective.

Ainsi se créèrent au fil des siècles toutes les figures de la dictature du visible.

\section{B) Ordinateurs et liberté}

Qu'en est-il de ce qu'on nomme les nouvelles images produites par les nouvelles techniques, c'est-à-dire par l'électronique?

Tout d'abord disons qu'elles ne sont plus si jeunes et que les ordinateurs ont des ancêtres qui voient leur descendance se multiplier. On parle d'ailleurs de génération. En vérité, on les appelle nouvelles non pas parce qu'elles sont jeunes mais parce qu'elles mettent en scène une rupture des générations parmi les citoyens. La jeunesse est à l'aise dans ce que les gens plus vieux apprennent avec difficulté. Comment assurer la transmission des symboles et de la mémoire, comment construire du sens quand ceux qui traditionnellement étaient demandeurs, maîtrisent mieux que leurs parents et que leurs maîtres l'instrument le plus performant de la transmission contemporaine? Quelque chose comme un renversement des instances de savoir a lieu puisque ceux qui ont tout à apprendre sont à leur façon des experts. La création comme la transmission du savoir passent par des outils non seulement complexes mais en continuelle mutation. Le temps passe, l'électronique envahit tout et nous nous demandons quel monde encore sensible elles vont construire, monde 
dans lequel il semble qu'un certain nombre d'entre nous s'affolent ou se sentent menacés. II faut que ceux qui veulent construire la mémoire et la citoyenneté non seulement apprennent à se servir des nouveaux outils, mais qu'ils y trouvent les moyens de mobiliser la pensée. En un mot, entre la machine et le programme, comment maintenir la place du tiers, le lieu de la parole? Remarquons d'entrée de jeu que tout ce qui passe par un ordinateur est soumis à un traitement binaire qui en fait un langage. Or un langage étant différent de la parole, la question est donc bien de savoir quelles sont les chances de l'incarnation d'une altérité et de la parole dans de tels dispositifs.

Je distinguerai deux aspects de la question. D'un côté les images de synthèse, images proprement numériques, de l'autre les effets généralisés de la numérisation grâce au traitement numérique de toutes les données, quelle qu'en soit l'origine analogique ou non, quelle qu'en soit la finalité artistique, informative ou scientifique.

\section{1) Le vertige numérique, le fantasme de «l'image juste»}

En premier lieu, remarquons que les images issues des seuls calculs numériques posent un problème singulier puisqu'elles se présentent à nous, avec tous les caractères antinomiques de limage. Elles sortent de mains savantes qui nous les proposent comme des produits propres, purs et vrais. Je veux dire que les images produites par des machines à partir de programmes numériques, à partir de modèles mathématiques et dans un langage binaire, se réclament d'un savoir sans ombre et sans erreur, sans le moindre écart substantiel avec leur modèle. Le traitement du hasard et la numérisation de ce que les experts ont étrangement nommé le "chaos", ne permettent à aucun moment de reconnaître un écart infranchissable entre ce qu'on voit et ce qu'on ne voit pas. On passe seulement d'un mode d'apparition à un autre, sans perte, sans bruit et sans déchet. Le calcul qui préside à la naissance du visible le revêt d'une étrange autorité quasiment néopythagoricienne, ou néoleibnizienne : le visible est le fruit de la science du nombre et toute la nature partage l'être des nombres. Image sans mélange, sans vie et sans mortalité, où l'aléatoire fait l'objet d'une prévision, fût-elle infinie. Ces visibilités provoquent une jouissance, celle de s'approprier les simulacres d'une substance intelligible. Cette jouissance est aussi celle de l'omnipotence, car la manipulation numérique n'a pas d'autre limite que celle des programmes eux-mêmes. Nous voici face à des visibilités qui remplissent toutes les conditions requises par les icono- 
clastes et iconophobes : pureté et vérité. Tout le problème de nos inquiétudes est là, puisqu'on peut se demander où est l'image telle que nous l'avons définie dans ces visibilités. On peut le dire autrement et demander de façon plus dynamique à ces images si elles renoncent au pouvoir d'incarner ou si au contraire elles nous mettent en charge de découvrir les nouvelles figures de l'incarnation. Face à des images exactes, nous cherchons la place de notre chair comme si ces visibilités étaient exsangues. Les mathématiques produisent un modèle tout puissant et apodictique où disparaît la part de l'ombre pour reprendre le mot de René Char «Nombre l'ombre niée». Comment reconquérir la part de l'ombre, la chair de l'absence, la parole au cœur des langages?

La dimension solaire et irréfutable est nécessairement fascinante pour le pouvoir qui peut rêver que tout débat soit suspendu devant l'évidence sans contradiction des visibilités nouvelles. L'image disparaît et avec elle toute la liberté que donnait son incertitude. On s'alarme, on dit que les images nous trompent, que les techniques simulent et nous tuent. On prend le deuil de l'altérité.

Les iconophiles avaient raison, il n'y a que les idolâtres qui croient aux images et qui les détruisent comme des personnes néfastes!

Erreur sur l'image elle-même. La question est celle de la croyance en la présence réelle qui est la conséquence idéologique du modèle mathématique, matrice du visible. L'image née d'un savoir, qui plus est partagé par un très petit nombre, jouit à la fois des honneurs dus à la science et du respect médusé des usagers. Si l'image née du savoir donne à croire, alors nous nous demandons si elle donne encore à penser.

L'absence d'écart, l'absence d'absence, la présence absolue du modèle incorruptible dans le champ du visible, met le spectateur dans une situation sacramentelle absolument semblable à celle qu'impose l'eucharistie. Par l'effet d'une mystérieuse alchimie, ce que l'on voit ne fait qu'un avec ce que l'on ne voit pas, tout comme ce que l'on mange ne fait qu'un avec ce que l'on devient. La vérité calculée du modèle favorise le retour d'un vieux fantasme théologique et métaphysique : il y aurait des images vraies! Et nous avons tous entendu l'enthousiasme naïf des ingénieurs du virtuel. En effet, l'équation modélisant et l'exactitude mathématique ayant valeur de vérité, il n'y a plus d'erreur, plus d'incertitude. Enfin de l'image pure, purifiée de toute altérité. Le corps de l'image rejoint le corps de la science et sa consommation rend tout ce qui participe à cette moderne sainte table, 
membre d'un corps universel, objet d'une digestion mondiale. Mais pourquoi cette situation ne serait pas considérée à l'inverse comme l'une des plus stimulantes qui ait été donnée aux consommateurs des imageries? Et si le malaise de l'exsangue mobilisait l'exigence d'altérité?

Donc la question est la suivante : les images de synthèses ontelles la possibilité d'incarner? La réponse est dans la question puisque la puissance de liberté contenue dans l'incarnation est appendue au regard et non à l'objet. L'absence de modèle réel et l'équivalence du langage visuel au langage mathématique ne laissent rien présumer des relations de ces visibilités avec la parole vivante. Une nativité n'est guère plus réelle qu'une équation. Une image n'a jamais tiré sa force de l'autorité de son modèle mais toujours des effets symboliques, donc de l'émotion et du jugement induit par sa présence d'image. Dire qu'une image est habitée par la parole est tout à fait différent du fait de reconnaître en elle l'effet visuel d'un langage. Benoît Mandelbrot, inventeur génial des grandeurs fractales et des visualisations du "chaos", donne une réponse remarquable : les images fractales quand elles sont des images donnent à rêver au même titre que toute autre image.

Mandelbrot rend sa totale liberté à l'image par rapport au savoir mathématique et reconnaît que les images fractales ont pour lui une séduction poétique. Cependant, son discours est une stupéfiante naïveté quand il aborde ce qu'il nomme l'esthétique. II fait entendre clairement que la pureté est un fantasme philosophique qui ne peut en aucun cas reprendre ses droits pour des raisons scientifiques. Maintenant, voyons de plus près ce qu'il appelle l'art du chaos. "Le coucher de soleil sur une île fractale", qui décore fièrement la couverture du livre, est une image née d'un langage. Où est la parole? C'est à nous de faire le choix entre l'idolâtrie de l'intelligible et la chair bruissante de toute visibilité. Pour Mandelbrot, cette image n'est pas loin d'être une œuvre d'art, un objet de dégustation. Disons qu'elle a une puissance ludique et onirique qui la met déjà dans un régime autre que celui de son modèle. Soit. Ce qui cloche, si je puis dire, c'est que Mandelbrot se situe dans la jouissance esthétique d'une représentation romantique de la nature simulée. Cette image est à son goût. Son fameux coucher de soleil est en vérité un chromo traditionnel digne du calendrier des postes. Ce révolutionnaire de la science ne conçoit pas que la charge du visible dans l'image n'est pas de l'ordre de la beauté comme esthétisation du réel mais de la mobilisation de la pensée. Les nouvelles images sont totalement 
disponibles à la création d'un sens, mais encore faut-il que ceux qui s'en chargent ne le fassent pas à titre d'experts scientifiques de l'illusion ou d'ingénieurs d'un nouvel art déco. L'avant-garde technologique et scientifique est totalement régressive voire même réactionnaire en matière de création. Les artistes et eux seuls peuvent trouver les formes et les figures de l'image dans les nouvelles visibilités, donc les créateurs vidéastes et cinéastes. Or ces créateurslà ne se trouvent pas confrontés à la résistance du monde scientifique, qui pourrait difficilement les empêcher d'inventer mais à celle du marché financier de la communication. C'est sur les écrans, leurs coûts et leurs usages "communicationnels", que la guerre se livre et la guerre des écrans est bien la nouvelle guerre des images contre les idoles. Les visibilités tuent l'image, le vieux combat reprend entre les tenants de l'incarnation et de la parole, et ceux de l'incorporation, bien sûr, sont très séduits par les couchers de soleil numériques et le bombardement des effets spéciaux. Ça ne dérange personne et ça divertit beaucoup. L'esthétique dite du chaos est le Disneyland de l'informatique. Dans le commerce du visible, il peut aussi arriver à la liberté du scientifique d'être menacée.

En vérité, rien n'empêche un créateur de mettre les images de synthèse au service d'un sens et d'une liberté du regard. Mais cela suppose deux choses : la maîtrise de l'outil, l'autonomie dans ses usages et la responsabilité éthique dans l'invention des nouveaux regards. Sur des images nouvelles il faut un regard nouveau.

C'est pourquoi j'examinerai pour finir deux cas spécifiques et récents d'une manipulation des images par "computer" par deux créateurs, disons plutôt par deux cinéastes, car c'est dans l'examen de ce qu'ils ont fait que nous saurons lequel est créateur. Cela nous conduira au cœur du problème, car c'est vraiment de responsabilité et de place du spectateur qu'il sera question.

\section{2) La manipulation numérique de l'archive}

Tout autre en effet est la question que pose l'utilisation généralisée des instruments électroniques dans la production du visible et de l'audible. Si nous sommes digérés par la nourriture que nous absorbons, nous pouvons aussi choisir de pas l'être et de demander aux producteurs d'images la part de l'ombre qui préserve notre place. Interroger les nouvelles technologies de l'image en terme de liberté est exactement la question qui se pose non pas aux machines mais à ceux qui en usent à la télévision, dans les media et dans l'art. Dire que la manipulation numérique des images nous conduit à ne 
plus croire à ce qu'on nous montre est la meilleure chose qui pouvait arriver. Cela signifie que la nature des images contemporaines a soudain ouvert les yeux sur le fait que les images ne sont rien d'autre que des propositions instables et indécises, qui n'ont ni substance ni vérité en dehors de la parole qui les habite et de la place faite à ceux qui les regardent. Les visibilités attendent de nous leur poids de sens. Former et construire le regard dès l'enfance non pour lui dire ce qui est vrai ou bon, mais pour reconnaître dans ce qu'on voit ce qui donne la parole et ouvre le débat, voilà ce qu'on peut attendre des nouveaux imagiers.

Donc, sans rejoindre le chœur des idolâtres de la substance vraie et pure des nouveaux simulacres, je pense cependant que la liberté de manipulation qu'ils inaugurent n'a jamais autant engagé politiquement ceux qui les fabriquent. Godard a dit qu'un travelling est une question éthique. On peut généraliser son propos et dire qu'aujourd'hui toute visibilité porte la signature d'un choix puisque le montage et la fabrication sont de part en part dans les mains responsables de ce qu'elles décident de montrer. Jamais les visibilités n'ont avec autant de force convoqué la pensée, précisément parce qu'elle est en danger. Plus l'incorporation s'impose sur le marché, plus s'élèvent les voix qui réclament le sens et la liberté. Nous en sommes la preuve aujourd'hui même. Je prendrai pour illustrer mon propos deux exemples qui feront conclusion. II s'agit de deux films, faits par deux réalisateurs qui l'un et l'autre ont eu recours aux effets les plus sophistiqués des nouvelles technologies de l'image. L'un est Eyal Sivan, l'autre Chris Marker. L'un et l'autre posent dans la production de leur objet la question de la vérité dans l'écriture de I'histoire et la manipulation numérique de l'archive. Dans les deux cas, il s'agit d'une enquête concernant deux des plus tragiques événements de notre siècle : l'extermination des juifs par les nazis et le suicide sur ordre des habitants de l'île d'Okinawa. Deux massacres liés à la dernière guerre.

Avec les meilleures intentions du monde, Sivan, dans Un Spécialiste, manipula les archives d'un procès pour en faire une fiction. Arendt servit de point de départ, nous dit-il, et devint ainsi garante de la hauteur des intentions. Malheureusement, l'erreur fut de considérer l'image comme l'équivalent d'un texte critique, avec sa démonstration et son interprétation des faits. Les auteurs du film, convaincus par le texte de la philosophe et lui accordant une valeur de vérité, les images qu'ils firent en son nom se trouvèrent, de ce fait, en demeure de transmettre une vérité. Ce que le texte de Hannah Arendt 
pouvait faire par la force démonstrative du discours critique, l'image ne pouvait s'en charger, comme si on ne faisait que changer de langage. Donner la parole à l'histoire par la voie des images nécessite qu'on change totalement de dispositif. On ne change pas de langage comme dans le cas des fractals, mais de régime dans la mise en commun d'une visibilité critique. Quand le lisible et le visible sont identifiés, on entre dans la communication et le programme informatif. L'émotion que cherche le Spécialiste ne quête que l'adhésion, et la pensée est réduite au silence. Pour que l'image mobilise les esprits et la liberté du jugement, il faut qu'elle soit aussi peu chargée d'intentions et de convictions que possible, comme une image de Resnais ou de Lanzmann mettant le spectateur en demeure de devenir le témoin responsable à son tour d'une mémoire. Ils ne décident pas à notre place de nos choix, mais construisent cette place. Sivan et Braumann diffusent leur doctrine, chose que n'a jamais faite Arendt!

Sans entrer dans le débat sur le fond concernant les Judenrat, on peut dire qu'il y eut là une décision très lourde puisque le spectateur est maintenu dans l'ignorance de ce qui sépare le document de la fiction et que les visibilités ont pris un parti. Le documentaire pose un problème spécifique puisqu'il y a une combinaison problématique de la présence réelle, indicielle si l'on veut, de ce qui est montré et qui a eu lieu, et que dans le même mouvement, on décide d'exploiter l'archive dans le sens d'une interprétation orientée. Or l'archive de départ était une image, rien de plus, en ce sens qu'elle laissait le spectateur libre de composer avec les ombres saisies à un moment de l'histoire et d'être témoin... Seule la parole donne ses chances à la liberté de penser. À l'époque, le speaker de la télévision américaine se chargeait de faire voir à l'Amérique ce qui "convenait". Arendt, elle, a dit et écrit ce qu'elle a vu avec son regard de philosophe, en tant que témoin c'est-à-dire ce qui restait invisible pour la télévision américaine. Son écriture nous rend témoins à notre tour, nous prend à témoin de ce qui n'était pas visible, sa pensée. Elle a proposé son opinion, elle a livré sa lecture au débat et l'on sait à quel point cette lecture prenait des risques, a fait problème et lui a valu la réprobation d'une partie de l'intelligentsia. Que reste-t-il de ce risque de la parole libre sur le visible dans un objet qui voudrait montrer par l'image la vérité d'une idée? La vérité du point de vue d'Arendt n'est pas dans ce qu'elle a vu, mais dans ce qu'elle a choisi de faire comprendre et de démontrer. Le discours construit le décryptage de ce qu'on ne voit pas, l'image, elle, construit la liberté d'un choix pour ce regard. La 
même image a des lectures contradictoires. Désormais, ce film existe et donne dangereusement l'exemple d'un droit de manipuler le regard dans le sens d'une idée, en trahissant non pas la réalité visible mais la fiction elle-même. Abandonner la fiction, c'est abandonner l'image au sens où je l'ai définie, en la distinguant des visibilités. Que pourront faire les révisionnistes avec les mêmes images, grâce aux mêmes procédés, en s'appuyant sur un autre livre ! Jamais l'image ne viendra les départager au nom de sa vérité, elle n'en a pas. Les nouvelles technologies de l'image ne peuvent se charger de dire le vrai à notre place. La construction du regard critique demande que l'image reste fidèle à l'incarnation d'une ambivalence indécidée a priori, qui attend qu'une parole réponde de son sens. C'est cette place qu'il faut sauver. Voilà pourquoi les films de Resnais ou de Lanzmann que je citais, sur les camps, ont choisi de n'imposer aucune démonstration autoritaire d'une vérité, ni l'insistance terrifiante de l'insoutenable prélevé dans la réalité. Ils ne font pas la preuve par l'image, mais ils nous mettent à l'épreuve de l'image. Leur vertu vient non de la vérité de l'image qui, elle, n'existera jamais, mais de la place spécifique qu'ils font aux spectateurs en leur laissant la responsabilité de leur jugement et de leur choix. Cette question fondamentale de la place du tiers dans la construction d'un objet offert à la pensée, c'est-à-dire à la parole échangée, se retrouve d'ailleurs à tous les niveaux des usages des nouveaux media. Que ce soit dans la pédagogie, où se pose la question de la relation du maître à l'élève, dans la médecine, celle de la relation du médecin au malade, dans la catéchèse ou le culte télévisé, où c'est la question de la coprésence des fidèles les uns aux autres, etc. Le problème revient inlassablement : que deviennent les relations des sujets de la parole dans la consommation des images? J'ai répondu à ma façon en disant que la machine est dans nos mains et que l'exigence maintenue d'une parole est au cœur de l'image.

C'est pourquoi je voudrais évoquer la démarche de Chris Marker dans Level Five. II use de tous les ressorts des jeux vidéo, de la manipulation numérique et de la création d'un site Internet pour produire de l'image. II fait appel au web, aux archives, aux figures virtuelles, aux images cloniques, tout notre monde est là pour raconter la transmission d'une histoire de guerre et d'une histoire d'amour sur le même mode, celui de l'impossibilité d'incorporer le vrai sur un écran, celui d'incarner la parole, dans l'inquiétude toujours présente de la vie et du brouillage. II construit l'inclusion de deux écrans, l'un qu'on pourrait appeler "réel» par commodité et qui n'est autre que 
celui de la fiction, une histoire d'amour, l'autre qui fait appel à toutes les ressources du virtuel et qui prend en charge la réalité historique. Cette réalité, c'est le suicide collectif et programmé des habitants d'Okinawa lors de la guerre nippo-américaine. Chris Marker mène son enquête sous forme de jeu vidéo. L'histoire d'amour engage d'emblée le visible dans la chair passionnelle de nos affects. Travailler l'image est toujours une aventure affective. La gestion éthique de nos affects est l'enjeu politique de nos images. Que choisissons-nous d'aimer et de hair ensemble? Laura incarne la voix d'une parole maintenue d'un bout à l'autre, et elle renforce la puissance du montage virtuel. La hauteur de ce film vient de ce qu'il arrive à problématiser complètement les effets de la manipulation. Nous sommes pris à témoin d'un bruissement de l'histoire avec les voix, les ombres croisées des vivants et des morts. Images impures, loin des certitudes indolores et de l'impérialisme du vrai, elles entretiennent avec nous un rapport de provocation dramatique et de mobilisation politique. Hybridation charnelle du réel et de la fiction. D'un bout à l'autre, nous sommes atteints par les effets d'une voix et d'un regard tendus vers un internaute fictif et qui s'adresse réellement à nous parce que nous lui faisons face sans le voir. Ce film est une démonstration spectaculaire des pouvoirs critiques qui habitent toute image digne de ce nom, quelles que soient les modalités de sa production technique. Le réalisateur pose directement le problème de la manipulation de l'archive et de la responsabilité vitale des faiseurs d'images. Une image peut tuer.

Jean-Luc Godard l'a montré et énoncé clairement dans Histoire(s) du cinéma, qui par la vidéo et le montage de l'image, de la voix et du son, déploient toute la force critique des images offertes au sens et font appel à la responsabilité historique et visuelle des spectateurs. L'image n'est qu'un passage pour la parole, C'est sa fragilité même et son inconsistance fondamentale qui en font justement pour nous une affaire grave. Les machines doivent nous faire parler et elles le peuvent. La liberté est toujours à la merci de nos mains. Encore faut-il ne pas faire monter l'image dans le train de la pureté, de la vérité et de la communication.

Je conclurai que tant qu'il y a des images il y a de la liberté, et l'espoir démocratique trouve la chair de sa parole dans des figures ouvertes à tous les moyens sans exception.

Marie-José Mondzain 